\title{
Distribution and predictive factors of seizure types in 104 cases
}

\author{
V. A. LACOMBE*, M. MAYES ${ }^{\dagger}$, S. MOSSERI ${ }^{\ddagger}$, S. M. REED ${ }^{\S \#}$ and T. H. OU" \\ Center for Veterinary Health Sciences, Oklahoma State University, USA \\ ${ }^{\dagger}$ College of Pharmacy, The Ohio State University, USA \\ ‡Clinique Veterinaire Veronique Susini, Peyrolles en Provence, France \\ $\S$ Department of Veterinary Clinical Sciences, The Ohio State University, USA \\ "Rood \& Riddle Equine Hospital, Lexington, Kentucky, USA \\ "The University of Michigan Center for Global Health, USA \\ *Correspondence email: veronique.lacombe@okstate.edu; Received: 18.03.13; Accepted: 14.07.13 \\ S. Mosseri's present address is: Three Counties Equine Hospital, Ripple, Stratford Bridge, Gloucestershire GL20 6EY, UK.
}

\section{Summary}

Reasons for performing the study: Although many studies have been performed to classify seizures by type in man and small animals, a similar study in horses is lacking.

Objectives: The aims of this study were as follows: 1) to characterise the distribution of seizure types in 104 horses presented for seizure disorders to a referral veterinary hospital; and 2) to characterise the various types of seizures by identifying associated clinical factors.

Study design: Retrospective analysis of clinical records.

Methods: Seizures were classified based on seizure type, according to the most recent accepted definitions in both human and small animal epileptology. History, clinical and neurological examinations, diagnostic investigations and post mortem examinations, when available, were recorded for univariable and multivariable logistic regression analyses.

Results: Seizures were categorised as primary generalised in $23 \%$ of horses, focal without secondary generalisation in $42 \%$ of cases and secondary generalised in $24 \%$ of cases. The type of seizure could not be classified in $11 \%$ of cases. Significant associations were found between seizure type and the following characteristics: 1) gender; 2) frequency of seizures; and 3) presence of seizures during hospitalisation. Seizure type was not significantly associated with aetiology. For a horse with recurrent seizures, the odds of having focal seizures was 3.7 times higher $(P=0.02)$ than in a similar horse with nonrecurring seizures in the final logistic regression model.

Conclusions: The majority of the seizures described were focal seizures with or without secondary generalisation. The clinical presentation was independent of the underlying disease.

Keywords: horse; generalised seizure; focal seizure; epilepsy

\section{Introduction}

Epileptic seizures are clinical manifestations of rapid excessive and/or hypersynchronous abnormal neuronal activity from the cerebral cortex that result in voluntary alterations of motor activity, consciousness, autonomic or sensory functions [1-3]. The Commission on Classification and Terminology of the International League Against Epilepsy (ILAE) created a comprehensive classification of seizures in man over 30 years [4]. A standardised classification has numerous advantages, including the foundation for a coherent and systematic approach to the diagnosis and treatment of epileptic seizures and for a common mode of communication among clinicians [4]. This ILAE nomenclature is based on a comprehensive diagnostic scheme organised in 5 axes, which includes phenomenology (defined as the description of the event; axis 1), type of seizures (axis 2) and aetiology of seizures (axis 3). Axis 1 consists of a description of the ictal semiology (defined as the signs and symptoms associated with seizures), including the pre- and postictal periods, during which the behaviour of the patient should be observed whenever possible. Axis 2 consists of the classification of seizures by type (focal vs. generalised). Focal seizures, which are caused by focal abnormal discharge, result in localised motor signs or sensations, the first clinical signs being indicative of the epileptic focus localisation $[1,3,5]$. Focal seizures were previously referred to as partial, a term that received criticism as it implies part of a seizure rather than a seizure that begins in part of one hemisphere $[5,6]$. Generalised seizures involve the entire cerebral cortex and result in generalised bilateral motor activity over the whole body, including convulsive seizures, nonconvulsive seizures and myoclonic seizures. A comprehensive classification of seizures in dogs and cats has been described based on a similar diagnostic scheme [7,8]. In addition, classification of seizures by aetiology (based on the results of diagnostic investigations and post mortem examinations) has recently been reported in horses [4]. However, to our knowledge, a classification of seizures by type (i.e. generalised vs. focal) has not been performed in horses. The purpose of this study was to classify seizures in horses by type using the diagnostic scheme proposed in man and small animals, and to determine the clinical characteristics of focal and generalised seizures.

\section{Materials and methods}

\section{Case selection and procedures}

Horses (of any age except neonates $<3$ weeks old) were presented to a referral veterinary teaching hospital with a primary complaint of seizure disorders from 1988 to 2009. Data extracted from medical records included case details, age, duration and frequency of the seizures, as well as the results of the clinical/neurological examinations and haematological and serum biochemical analyses. Data were further extracted from the results of the ancillary diagnostic tests, including electroencephalogram recordings, cerebrospinal fluid analysis, skull radiographs and computed tomography (CT) imaging of the head, as well as gross and histopathological examinations of the central nervous system when available.

\section{Classification of seizures}

Seizures were classified based on seizure phenomenology (axis 1) and seizure type (axis 2) according to the most recent accepted definitions/guidelines in small animal and human epileptology $[6,7,9,10]$. Seizures were classified based on historical information, follow-up telephone communications with referring veterinarians, and evaluation 


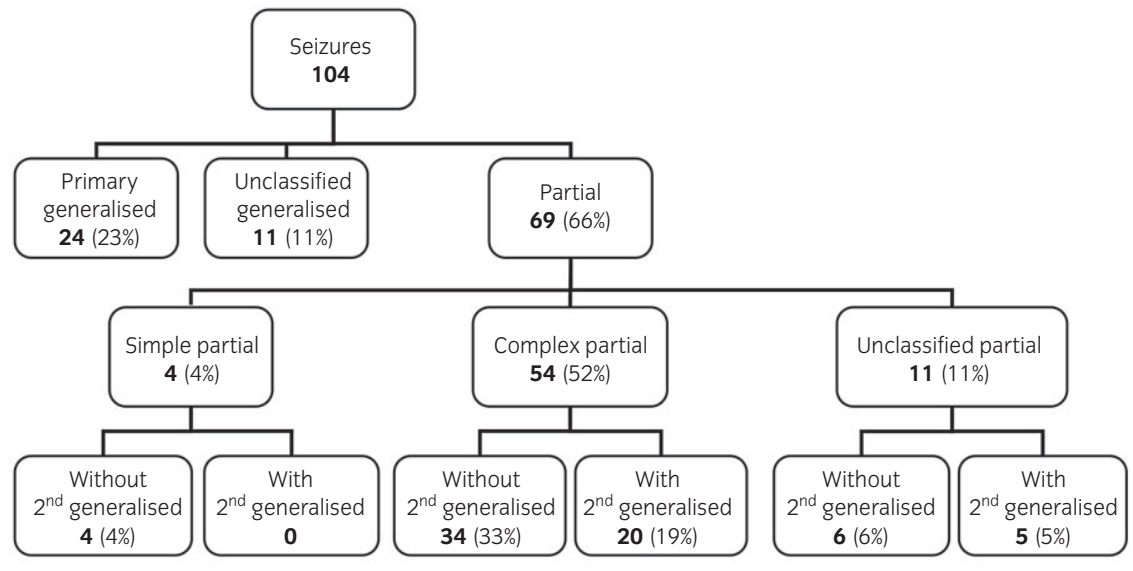

Fig 1: Classification of seizures by type in horses (axis 2 of the International League Against Epilepsy diagnostic scheme). of seizures by at least one clinician when they occurred during hospitalisation. Seizures were further grouped by frequency $(<2$ and $\geq 2$ episodes), which included all the episodes reported prior to referral and during hospitalisation. A cluster of seizures within $24 \mathrm{~h}$ was regarded as a single episode [1]. If normal mentation was not completely recovered between successive seizures within a period of $5 \mathrm{~min}$, the horse was considered to be in 'status epilepticus' [11]. Abnormal neurological signs (e.g. abnormal mentation or behaviour) during the pre-ictal, ictal and postictal periods were reported when available in the medical record or when observed during hospitalisation (axis 1). Seizures were categorised by type (axis 2) as focal or generalised. Seizures that involved a focal area of the cerebral cortex with resulting localised neurological signs were classified as focal seizures and were further categorised as follows: 1) simple, if alertness and normal mentation were maintained; or 2) complex, if impairment of consciousness was reported. Generalised seizures that originated from both cerebral hemispheres from the onset were referred to as primary generalised seizures. Generalised seizures that progressed from focal seizures were referred to as secondary generalised seizures. Seizures were further classified by aetiology as reactive (i.e. presence of a temporary systemic disease with normal brain function), symptomatic (i.e. presence of an identifiable structural brain lesion) or of unknown origin (i.e. cryptogenic) based on the results of the ancillary diagnostic tests, as previously described [4].

\section{Data analysis}

Data were categorised by cut-off values to facilitate logistic regression analysis as follows: breed, sex, age, frequency of seizures, history of head trauma, mentation, behaviour, cranial nerve and gait deficits, precipitating factors, occurrence of seizures during hospitalisation, peri-ictal events, electroencephalogram, cerebrospinal fluid analysis, CT imaging of the head, skull radiographs, blood analysis, post mortem examination of the central nervous system and aetiological classification. Crude odds ratios (ORs) were used to measure the association between each independent variable and the outcome of interest, and were determined by univariable analysis. All the variables significantly $(P<0.05)$ associated with type of seizure in the univariable analysis were included in the final logistic regression model. Values $\mathrm{P}<0.05$ were considered statistically significant. The Hosmer-Lemeshow goodness of fit test was determined to assess model fit, an insignificant test indicating that the model fits the data well. Data analysis was conducted using SAS software (version 9.2) .

\section{Results}

One hundred and four horses presented for seizure disorders or developed seizures during hospitalisation ( 88 and 16 horses, respectively). Forty-two (40.4\%) horses with seizure disorders were females, 19 (18.3\%) were males and 43 (41.3\%) were geldings, with 14 breeds represented and with ages ranging between 1 month and 30 years on presentation, with a median age of 10 years.

\section{Classification and seizure characteristics}

Seizures were classified according to their types, primarily from the history and the clinical observation in the hospital. Sufficient information regarding ictal phenomenology was available to classify seizures by type in 82 (79\%) cases (Fig 1). Twenty-four horses (23\%) had primary generalised and 69 (66\%) had focal seizures (with or without generalisation). The onset of the generalised seizure episode was not observed in $11(11 \%)$ cases, which remain unclassified. Among horses with focal seizures, 54 (52\%) horses had complex focal seizures with or without generalisation (20 and 34, respectively). None of the horses affected with simple focal seizures (4) developed generalised seizures. Impairment of consciousness could not be established in 11 horses with focal seizures, for which a classification could be reached only partially. When including these cases, 25 (24\%) horses had secondary generalised seizures.

Overall, $44(42 \%)$ horses had focal seizures without generalisation, and $60(58 \%)$ horses had primary or secondary generalised seizures. The most commonly reported focal seizure phenomenology included motor signs or sensations involving the face (e.g. ear or lip twitching, tongue prolapse and salivation, tonic opening or closing of the jaw, compulsive biting, licking, chewing or rubbing of the face, tremors in the chin and nostrils or violent headshaking) or the body (e.g. compulsive kicking, tremors, lifting one limb, going down on the knees, dog-sitting or head turning to one side). More complex behaviour patterns with impaired consciousness and behavioural changes were also reported, including hyper- or hyporesponsiveness to external stimuli, increased avoidance of the menace test, disorientation or sudden intermittent aggression. Localised tremors progressing to the limbs and to the ipsilateral side of the body, evoking the clinical presentation of Jacksonian seizures, were also described. Generalised seizures were described as tonic-clonic motor activity, with eye globe deviation or horizontal/vertical nystagmus, generalised rigidity or loss of muscle tone. Two horses were showing abnormal mentation and facial twitching for $>20 \mathrm{~min}$, and were considered to be in status epilepticus. Vocalisation and/or autonomic disturbances (mydriasis, uncontrolled urination or defaecation or profuse sweating) were also reported.

Prodromes were described in 13 horses affected by focal or generalised seizures and included isolation from other horses in the field, anxious behaviour, standing in a corner of the stall, compulsive swishing of the tail or acting in a more friendly manner than usual towards the caretaker. The postictal period, which lasted from minutes to hours, was documented in $32(31 \%)$ horses affected by complex focal or generalised seizures. Postictal signs included depression, ataxia, disorientation, hypersensitivity or agitation, shallow breathing and blindness. Precipitating factors were reported in $11(11 \%)$ horses affected by focal and generalised seizures, and included sedative agents (e.g. xylazine), noise/sound, trimming the mane, touching the neck area or oestrus.

Based on the ancillary diagnostic tests and/or post mortem examination (when available), an underlying structural brain lesion (i.e. symptomatic seizures) was identified in 15 and 27 horses exhibiting focal and (primary or secondary) generalised seizures, respectively. Findings included skull 
TABLE 1: Multivariable analysis of predictive factors for generalised seizures

\begin{tabular}{|c|c|c|c|c|c|}
\hline Predictive factor & & Wald Chi-square & Odds ratio & 95\% Confidence interval & $P$ value \\
\hline \multirow[t]{3}{*}{ Gender } & Gelding & & 1.00 & Reference & \\
\hline & Male & 0.07 & 1.18 & $0.37-3.79$ & 0.785 \\
\hline & Female & 2.95 & 2.28 & $0.89-5.82$ & 0.086 \\
\hline \multirow[t]{2}{*}{ Frequency of seizures } & 1 episode & & 1.00 & Reference & \\
\hline & $\geq 2$ episodes & 5.52 & 0.27 & $0.09-0.81$ & $0.019 *$ \\
\hline \multirow[t]{2}{*}{ Seizure during hospitalisation } & No & & 1.00 & Reference & \\
\hline & Yes & 0.35 & 1.31 & $0.53-3.23$ & 0.556 \\
\hline
\end{tabular}

$\mathrm{n}=104$. ${ }^{*}$ ssing $\mathrm{P}<0.05$ as a criterion for inclusion of the variables in the multivariable model. Goodness-of-fit statistics: $r^{2}=0.12 ;$ maximal rescaled $r^{2}=0.16$. Hosmer-Lemeshow goodness-of-fit test: Chi-square $=2.19$, d.f. $=6$ and $\mathrm{P}=0.901$ (insignificant test indicates that the model fits the data well); $\mathrm{c}$ (area under the receiver operating characteristic curve) $=0.688$

fractures, cerebral haemorrhage, cerebral oedema, neoplasia (e.g. diffuse oligodendroglioma, extensive nasal adenocarcinoma with invasion to the cranial vault, brain lymphoma or pituitary adenoma), cholesterinic granuloma, vasculitis, meningo-encephalitis, abscesses, intracranial vascular events, leukoenphalomalacia, congenital abnormalities and equine protozoal myeloencephalitis. As a result of unremarkable findings on clinical evaluation, seizures of unknown origin were reported in 27 and 26 horses exhibiting generalised and focal seizures, respectively, including all the horses affected with simple focal seizures. Reactive seizures were identified in 2 horses with generalised seizures, secondary to liver failure and to severe acute haemorrhage. A genetic predisposition was suspected in 2 Arabian foals affected by secondary generalised seizures that exhibited unremarkable findings on clinical evaluation. An aetiological classification of seizures could not be established in 5 horses because of a partial clinical evaluation.

\section{Clinical factors associated with focal and generalised seizures}

Based on the univariable logistic analysis, no association between age, breed and the type of seizures was found within our study population. Females were more likely to suffer from (primary or secondary) generalised seizures than geldings $(\mathrm{OR}=2.6,95 \%$ confidence interval $[\mathrm{Cl}] 1.06-6.23, \mathrm{P}=$ 0.037 ; Supporting Information Item S1). There was a tendency for horses with alterations in mentation secondary to head trauma to exhibit generalised seizures ( $\mathrm{OR}=6.6,95 \% \mathrm{Cl} 0.80-54.99, \mathrm{P}=0.08$ ). For a horse with recurrent seizures, the odds of having (primary or secondary) generalised seizures was 4.2 times lower than for a horse exhibiting a single episode $(\mathrm{OR}=0.24,95 \% \mathrm{Cl} 0.09-0.65, \mathrm{P}=0.005$; Supporting Information Item S1). There was a tendency for horses affected by (primary or secondary) generalized seizures to exhibit a gait deficit on interictal neurological examination compared with a horse exhibiting focal seizures $(\mathrm{OR}=2.1,95 \% \mathrm{Cl} 0.93-4.77, \mathrm{P}=0.07)$. Generalised seizures were twice as likely to be observed during hospitalisation than focal seizures without generalisation $(\mathrm{OR}=2.2,95 \% \mathrm{Cl} 1.00-4.92, \mathrm{P}=0.048$; Supporting Information Item 51). The presence of pre- and postictal events and precipitating factors was not significantly associated with seizure type. No significant association was found between ancillary diagnostic tests (i.e. blood chemistry profile, electroencephalogram recordings, cerebrospinal fluid analysis, skull radiograph and CT imaging), post mortem examinations and the distribution of seizure type.

In order to evaluate predictive factors while controlling for potential confounding factors, a multivariable logistic regression analysis was developed (Table 1). The 3 variables significantly associated with type of seizure in the univariable analysis (i.e. sex, observation of seizure during hospitalisation and frequency of seizures) were included in the final model. In addition, the association between these 3 selected variables was assessed using Pearson's correlation coefficient, which did not reveal any strong correlations. In the final logistic regression model, horses with recurrent seizures (i.e. epilepsy) were more likely to exhibit focal seizures than a similar horse exhibiting nonrecurring seizures (Table 1). The Hosmer-Lemeshow goodness-of-fit test indicated that the model fits the data well $(P=0.901)$.

\section{Discussion}

The diagnosis of seizures in horses can be very challenging and is further complicated by the disparities in terminology used by equine clinicians and in veterinary studies. In addition, it is common to refer to generalised seizures without indicating whether they are generalised from the onset (primary generalised) or secondary to a focal seizure (secondary generalised). Therefore, there is a need for a common terminology and comprehensive classification. Seizures were recently classified by their aetiology (axis 3 of the ILAE diagnostic scheme) in the same large (>100 cases) referral equine-based population, with the majority of seizures reported being symptomatic or of unknown origin (i.e. cryptogenic) [4]. However, this is the first attempt to characterise the distribution of seizure types (axis 2) in horses. The majority (66\%) of the seizures described were focal, with or without secondary generalisation, and $23 \%$ were primary generalised. This distribution of seizures is in agreement with studies in small animals and man. In dogs, it has been reported that primary generalised seizures account for less than a third of the reported seizures, and that focal seizures with or without secondary generalisation appear to be the most common type of seizure observed $[7,12,13]$. Likewise, focal seizures were the most common type of seizure in cats [8], as well as in human epileptic patients based on large epidemiological and meta-analysis studies [14-18]. In our study, $11 \%$ of the generalised seizures could not be classified between primary and secondary generalised seizures. Although seizure episodes in horses are much less likely to be observed from the onset because they are less attended, this percentage was similar to the ones reported in small animals $(3-6 \%$ of unclassified) $[7,12,13]$, as well as in man $[14,15,18]$. Therefore, when applying the ILAE definitions, similarities in regard to seizure phenomenology and type exist between horses, small animals and man.

A partial onset of seizure has been associated with the existence of underlying focal brain pathology, given adequate technology [3,7]. In our study, an underlying disorder was identified in only $37 \%$ (15 of 41 ) horses presented with focal seizures and 50\% (27 of 54) of horses with (primary or secondary) generalised seizures, which highlights the difficulty in diagnosis of seizures, in part due to the limited number of tools capable of imaging the adult equine brain. Indeed, CT imaging of the head identified an intracranial structural lesion in only $12 \%$ (2 of 17) and 35\% (8 of 23) of horses affected with focal and generalised seizures, respectively. Although CT is also a sensitive diagnostic method for the detection of skull fractures, calcification and acute haemorrhage in the brain, it has limited diagnostic value to detect inflammatory disorders and small and/or diffuse parenchymal lesions $[19,20]$. Therefore, EEG and magnetic resonance imaging have mostly replaced the use of CT for the diagnosis of seizures in man and small animals, although a poor sensitivity in detecting epileptogenic areas in dogs with focal seizures has been reported [21]. Although EEG recordings revealed focal paroxysmal activity in 50\% (11 of 22) of the horses exhibiting focal seizures, no significant correlations could be established between EEG findings and seizure type in our study. Indeed, EEG changes are not pathognomonic of a disease, but rather reflect the kind of process occurring (e.g. acute vs. chronic; inflammatory vs. degenerative) [22]. In addition, the initial epileptogenic focus may rapidly progress to adjacent cerebral areas and potentially spread diffusely 
throughout the entire cortex, owing to a failure of local surrounding inhibitory zones to prevent the spread of the epileptogenic activity. Therefore, a focal seizure is usually caused by diffuse areas of cerebral dysfunction rather than by a small and well-delineated epileptogenic focus [5]. In addition, similar to previous reports [23], normal interictal EEG recording does not rule out the existence of seizures, because EEG recording was within normal limits in $13 \%$ (7 of 52) of cases presented with focal or generalised seizures. Indeed, the window of recording may not have been long enough to record interictal paroxysmal epileptiform discharges. To reduce the probability of false-negative recordings, frequent and prolonged EEG monitoring, such as a $24 \mathrm{~h}$ ambulatory EEG, is routinely performed in man, but this modality has not been used in horses [24]. Therefore, more advanced neuroimaging techniques are needed to increase the detection of focal structural brain lesions.

The second objective of this study was to identify clinical variables and predictive factors that were significantly associated with the type of seizure (focal vs. generalised). Seizure type was significantly associated with gender based on the univariable analysis, with females being more prone to generalised seizures. One mare demonstrated episodes of generalised seizures while being in oestrus. Oestrogen and progesterone levels have been shown to be related to alterations in seizure threshold in women and female dogs [12], and a similar risk factor appears to apply to equine seizures. In addition, seizure type was not associated with its aetiology (symptomatic vs. unknown cause), reflecting the fact that the clinical presentation of seizure is independent of the aetiology, but rather reflects the area and the extent of the cerebral cortex involved [25]. Consistent with our findings, the distribution of the various types of seizures was not associated with the underlying disease and the aetiological classification in both dogs and cats $[7,8]$. Furthermore, generalised seizures were more likely to be observed during hospitalisation than were focal seizures without generalisation. This finding highlights the fact that detailed clinical observation regarding the onset of seizure is required, because focal seizures that may be in the form of subtle and transient clinical signs may go undetected or be interpreted as the pre-ictal phase of an impending seizure [7]. Therefore, thorough historical investigation and clinical observation of the onset of seizures may increase the number of focal seizures identified in horses, as for other species [21], and would allow the clinician to differentiate further between primary and secondary generalised seizures. For a horse with recurrent seizures (i.e. epilepsy), the odds of exhibiting focal seizures was $\sim 4$ times higher than in a similar horse with one episode, in both the univariable and the multivariable modelling. This is in agreement with studies in human patients affected by epilepsy, which reported that focal seizures prevailed over generalised seizures [16-18]. A similar observation has recently been reported in veterinary medicine [7]. Although it was commonly believed that dogs with epilepsy were more likely to exhibit generalised seizures [26], it was demonstrated that when applying ILAE definitions (similar to the ones used in this study), focal seizures were more frequently associated with secondary/acquired (i.e. symptomatic and cryptogenic) epilepsy [7]. In addition, focal onset is now more frequently recognised in dogs and man affected with epilepsy of suspected genetic origin (i.e. primary and also previously referred to as idiopathic), which has usually been associated with primary generalised seizures [21]. Consistent with these findings, Arabian foals with epilepsy of suspected genetic origin in our study exhibited secondary generalised seizures. Likewise, although the majority of Arabian foals reported to have juvenile idiopathic epilepsy exhibited primary generalised seizures, some cases had focal onset episodes that became generalised secondarily [27]. Overall, the distribution of seizure types in horses and their predictive factors are similar to the ones reported in man and in small animals.

In summary, this study provided a comprehensive classification of seizures by type in a referral-based equine population and showed similarities between seizure semiology expressed in horses and other species. For instance, focal seizures were more prevalent than generalised seizures in our equine patients. In addition, our findings suggested that the clinical presentation was independent of the underlying disease and rather reflected the area and the extent of the cerebral cortex involved. Therefore, this classification could provide a tool for the clinician to understand seizure development better and to measure clinical outcomes objectively.

\section{Authors' declaration of interests}

No competing interests have been declared.

\section{Ethical animal research}

Ethical review not required by this journal: retrospective clinical study.

\section{Sources of funding}

None.

\section{Authorship}

V.A. Lacombe was responsible for study design and execution, data analysis and interpretation, and preparation of the manuscript. M. Mayes contributed to data collection and analysis; S. Mosseri contributed to data collection and manuscript preparation; S.M. Reed contributed to the majority of cases; and T.H. Ou contributed to data analysis.

\section{Manufacturer's address}

aSAS, Cary, North Carolina, USA

\section{References}

1. ILAE Commission on Epidemiology and Prognosis, International League Against Epilepsy (1993) Guidelines for epidemiologic studies on epilepsy. Epilepsia 34, 592-596.

2. Podell, M. (1996) Seizures in dogs. Vet. Clin. North Am. Small Anim. Pract. 26, 779-809.

3. Berendt, M., Gredal, H. and Alving, J. (2004) Characteristics and phenomenology of epileptic partial seizures in dogs; similarities with human seizure semiology. Epilepsy Res. 61, 167-173

4. Lacombe, V.A., Mayes, M., Mosseri, S., Reed, S.M., Fenner, W. and Ou, T.H. (2012) Epilepsy in horses: aetiological classification and predictive factors. Equine Vet. J. 44, 646-651.

5. Engel, J. Jr and International League Against Epilepsy (2001) A proposal diagnostic scheme for people with epileptic seizures and with epilepsy: report of the ILAE Task Force on classification and Terminology. Epilepsia 42, 796-803.

6. Commission on the Classification and Terminology of the International League against Epilepsy (1989) Proposal for revised classification of epilepsies and epileptic syndromes. Epilepsia 30, 389-399

7. Berendt, M. and Gram, L. (1999) Epilepsy and seizure classification in 63 dogs: a reappraisal of veterinary epilepsy terminology. J. Vet. Intern. Med. 13, 14-20.

8. Schriefl, S., Steinberg, T.A., Matiasek, K., Ossig, A., Fenske, N. and Fischer, A. (2008) Etiologic classification of seizures, signalment, clinical signs, and outcome in cats with seizure disorders: 91 cases (2000-2004). J. Am. Vet. Med. Assoc. 233, 1591-1597

9. Commission on the Classification and Terminology of the International League against Epilepsy (1981) Proposal for revised classification and electroencephalographic classification of epileptic seizures. Epilepsia 22, 489-501.

10. Podell, M. (2009) Differential diagnosis of seizures in dogs and cats. Proc SEVC Southern European Veterinary Conference, Barcelona.

11. Zimmerman, R., Hulsmeyer, V.I., Sauter-Louis, C. and Fischer, A. (2009) Status epilepticus and epileptic seizures in dogs. J. Vet. Intern. Med. 23, 970-976.

12. Berendt, M., Gredal, H., Pedersen, L.G., Alban, L. and Alving, J. (2002) A cross-sectional study of epilepsy in Danish Labrador Retrievers: prevalence and selected risk factors. J. Vet. Intern. Med. 16, 262-268.

13. Berendt, M., Gulløv, C.H., Christensen, S.L., Gudmundsdottir, H., Gredal, H., Fredholm, M. and Alban, L. (2008) Prevalence and characteristics of epilepsy in the Belgian shepherd variants Groenendael and Tervueren born in Denmark 1995-2004. Acta Vet. Scand. 50, 51.

14. Forsgren, L., Bucht, G., Eriksson, S. and Bergmark, L. (1996) Incidence and clinical characterization of unprovoked seizures in adults: a prospective population-based study. Epilepsia 37, 224-229. 
15. Keränen, T., Sillanpää, M. and Riekkinen, P.J. (1988) Distribution of seizure types in an epileptic population. Epilepsia 29, 1-7.

16. Kotsopoulos, I.A., van Merode, T., Kessels, F.G., de Krom, M.C. and Knottnerus, J.A. (2002) Systematic review and meta-analysis of incidence studies of epilepsy and unprovoked seizures. Epilepsia 43, 1402-1409.

17. Kotsopoulos, I., de Krom, M., Kessels, F., Lodder, J., Troost, J., Twellaar, M., van Merode, T. and Knottnerus, A. (2005) Incidence of epilepsy and predictive factors of epileptic and non-epileptic seizures. Seizure 14, 175-182.

18. Sander, J.W., Hart, Y.M., Johnson, A.L. and Shorvon, S.D. (1990) National General Practice Study of Epilepsy: newly diagnosed epileptic seizures in a general population. Lancet 336, 1267-1271

19. Sogaro-Robinson, C., Lacombe, V., Reed, S.M. and Balkrishnan, R. (2009) Factors predictive of abnormal results for computed tomography of the head in horses affected by neurologic disorders: 57 cases (2001-2007). J. Am. Vet. Med. Assoc. 235, 176-183.

20. Lacombe, V.A., Sogaro-Robinson, C. and Reed, S.M. (2010) Diagnostic utility of computed tomography imaging in equine intracranial conditions. Equine Vet. J. 42, 393-399.

21. Viitmaa, R., Cizinauskas, S., Bergamasco, L.A., Kuusela, E., Pascoe, P., Teppo, A.M., Jokinen, T.S., Kivisaari, L. and Snellman, M. (2006) Magnetic resonance imaging findings in Finnish Spitz dogs with focal epilepsy. J. Vet. Intern. Med. 20, 305-310

22. Lacombe, V.A. and Andrews, M. (2007) Electrodiagnostic evaluation of the nervous system. In: Equine Neurology, Eds: S.M. Reed and M. Furr, Blackwell, Oxford. pp 127-148.
23. Lacombe, V.A., Podell, M., Furr, M., Reed, S.M., Oglesbee, M.J., Hinchcliff, K.W. and Kohn, C.W. (2001) Diagnostic validity of electroencephalography in equine intracranial disorders. J. Vet. Intern. Med. 15, 385-393.

24. van der Ree, M. and Wijnberg, I. (2012) A review on epilepsy in the horse and the potential of ambulatory EEG as a diagnostic tool. Vet. Q. 32, 159167

25. Lacombe, V.A. (2010) Seizures and narcolepsy. In: Equine Internal Medicine, 3rd edn., Eds: S.M. Reed, W. Bayly, R.B. McEachern and D. Sellon, Saunders, St Louis, Missouri. pp 572-580.

26. Podell, M., Fenner, W.R. and Powers, J.D. (1995) Seizure classification in dogs from a nonreferral-based population. J. Am. Vet. Med. Assoc. 206, 1721-1728.

27. Aleman, M., Gray, L.C., Williams, D.C., Holliday, T.A., Madigan, J.E., LeCouteur, R.A. and Magdesian, K.G. (2006) Juvenile idiopathic epilepsy in Egyptian Arabian foals: 22 cases (1985-2005). J. Vet. Intern. Med. 20, 1443-1449.

\section{Supporting information}

Additional Supporting Information may be found in the online version of this article at the publisher's website:

Supporting Information Item S1: Results of univariable analysis of predictive factors for generalised seizures.

\section{EQUINE VETERINARY JOURNAL BOOKSHOP}

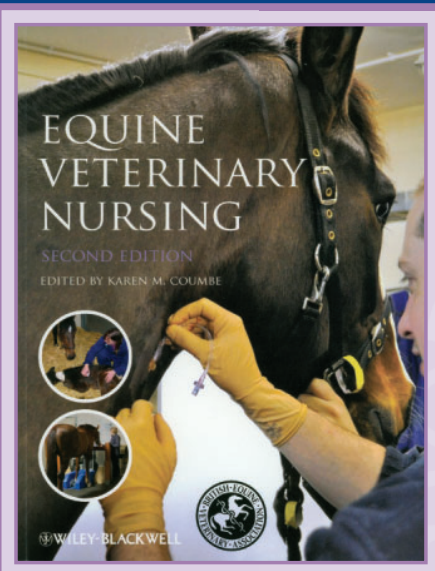

\section{Equine Veterinary Nursing Second Edition}

Edited by Karen M. Coumbe

Publisher: Wiley-Blackwell • August 2012 - Paperback, 496 pages

The breadth and depth of expertise essential for equine veterinary nursing today is provided in this definitive textbook. Thoroughly revised and updated in its second edition, and now fully illustrated in colour. This book covers the content of the everexpanding equine nursing syllabus, while still succinctly covering all the basics that veterinary nurses specialising in other species need to know. The key subjects included are: basic equine management, medical and surgical equine nursing, nutrition, reproduction and foal care, emergency first aid, and the theory underpinning these and other important topics.

Equine Veterinary Nursing Second Edition is presented in a clear and user-friendly manner, appropriate for all those involved in equine nursing. A practical approach is used throughout and procedures are illustrated with a large number of line diagrams and clinical photographs. It is an essential manual for all student and qualified equine veterinary nurses and all those involved in the care of horses.

Key Features: Includes a new chapter on the equine nurse's professional responsibilities - Major revisions to the chapters on diagnostic imaging and surgical nursing to reflect advances in technology and techniques • All information on health and safety management updated in keeping with new regulations • Authors include both vets and experienced equine nurses - Endorsed by the British Equine Veterinary Association.

EVJ Bookshop, Mulberry House, 31 Market Street, Fordham, Ely, Cambs. CB7 5LQ, UK Tel: $01638723555 \triangleleft$ Fax: $01638724043 \triangleleft$ Email: bookshop@evj.co.uk $\triangleleft$ www.beva.org.uk 